\title{
Efficiency of personal protective equipment used in agriculture
}

\author{
Luiz Antônio Meirelles ${ }^{\mathrm{a}^{*}}$, Marcelo Motta Veiga ${ }^{\mathbf{b}, \mathbf{c}}$ and Francisco José de Castro Moura Duarte ${ }^{\mathrm{a}}$ \\ ${ }^{a}$ Federal University of Rio de Janeiro, Av. Horácio Macedo, 2030, G-209, Prédio do Centro de Tecnologia, \\ Cidade Universitária, 21.941- \\ ${ }^{b}$ Strategic Management Department, Business School, Federal University of Rio de Janeiro State, Rua \\ Voluntários da Pátria, 107, Botafogo, Rio de Janeiro, Brazil, 22.270-010 \\ c 914Environmental Health Department, National School of Public Health, Oswaldo Cruz Foundation, Ministry of \\ Health, Rua Leopoldo Bulhões, 1480 / 503 - Manguinhos, 21.041-210 - Rio de Janeiro - RJ - BRASIL
}

\begin{abstract}
Despite the practical importance of Personal Protective Equipment (PPE), both in production and in consumption, it is observed a main problem regarding the quality of many PPE's used in Brazil. This study found a gap between the state of the art and the state of prevailing practices in the design of PPE's, which main consequences affect the health of workers who use PPE in agriculture activities. The results of this study identified legal constraints of this occurrence, involving the validation of PPE designed for isolate agents, without the analysis of relevant activities and without prototypes experiments in more frequent use situation
\end{abstract}

Keywords: personal protective equipment, agriculture, design

*Corresponding author. E-mail: meirelles@ufrj.br 


\section{Introduction}

This study examines the efficiency of personal protective equipment (PPE) used in agriculture throughout a theoretical framework, providing an analysis of PPE design as a possible solution to control unhealthful situations of rural workers.

The use of PPE, although the last preferred solution, must be considered within an integrated and systematic vision of occupational problems. For this reason, the efficiency of the entire occupational health and safety system is intimately related to the way a balanced choice of alternatives for prevention, protection and control is made [1].

Various problems can cause PPE to be inadequate for certain working conditions. Some desirable characteristics that were designed to provide greater occupational health and safety may also introduce operational difficulties in many work situations.

In Brazil this problem of the inadequacy of PPE to ergonomic and environmental conditions is particularly present in agriculture, where it is common to find farmworkers not wearing obligatory PPE while handling and applying agricultural chemicals. One of the reasons given is that many PPE items cause thermal discomfort, and in extreme cases can even lead to heat stress [2].

In France, recent studies $[3,4]$ demonstrated that PPE could not protect efficiently rural workers, and could even be the source of contamination. These studies indicated that rural workers could be contaminated while dressing or undressing their PPE and highlighted the inefficiency of PPE's permeability. These studies indicated that pesticides could easily penetrate through PPE cloth in a matter of minutes.

Another aggravating factor for the use of PPE by rural workers in countries with hot and humid climates is the fact that working under such conditions is more unhealthful than carrying out the same activities in more amenable conditions [5].

Personal protective items in the case of pesticides are designed to ensure protection against outside chemical agents, that is, to keep these substances away from the body. The same physical and chemical properties that provide this protective characteristic also often make the items very uncomfortable to wear.

The use of personal protective equipment is part of the daily routine of many workers. Many occupational health and safety projects contemplate the use of PPE from their conception. But by their nature, these protective items seek to reduce/control the risks to workers' health and safety, not to prevent them.
Preventive measures are those that eliminate/reduce risks at their source. They prevent/reduce the generation of the risk. Prevention should be prioritized over protective measures. However, in the majority of situations "protection" seems to be more "economical" than prevention.

For this reason, it is necessary for legislation to provide mechanisms that encourage prevention and stimulate collective protective measures by employers. Nevertheless, especially in real agricultural situations, these measures are often simply not utilized, for a number of reasons, among them high cost and design poorly adapted to the specific agricultural conditions.

This lack of stimulus for taking preventive measures to control unhealthful/hazardous conditions encourages the use of PPE as a palliative solution, since normally PPE appears to be the most economically advantageous solution.

Two indicators of this lack of incentives to preventive measures are the scarcity of studies on PPE's technological development and the assumption in Brazilian legislation that the use of PPE would be sufficient to avoid unhealthful conditions.

It can be inferred that the state of the art of PPE often fails to consider the use of these items in real situations. Many items of protective clothing are simply not suitable for their intended use. In the majority of cases these items do not manage to prevent contamination and sometimes could be even the source of contamination. The function of PPE becomes only to reduce the risk or mitigate the consequence, which diverges from the legal expectation. The possibility of contamination of farmworkers depends on the efficiency of the actual PPE.

\section{PPE - Personal Protective Equipment}

PPE's sale in Brazil has as main objective minimize any health damage resulted from the exposure to aggressive isolates agents. According to Law, the PPE must have a CA - Certificate of Approval, issued by the Ministry of Labor and Employment. In this selection of EPE's main function lies a problem - the preferred option for isolate agents seems to be raised to the statute of technical constraint for all PPE's projects. The relevance of this condition is in its national scope - many PPEs manufacturers adopt the sale for use in agriculture activity throughout the country national territory. 
The design of PPE oriented for particular agents is remarkable in the literature about the subject " Personal Protective Equipment ", especially in articles with a diagnostic character (Veiga, 2007), in patents related to PPE, and in standards for testing. An early example is the U.S. 170,942 patent, of an ear-like shell, in October 26th, 1875, to protect against cold (see Figure 1). The design practice is consistent with a significant part of the intellectual production.

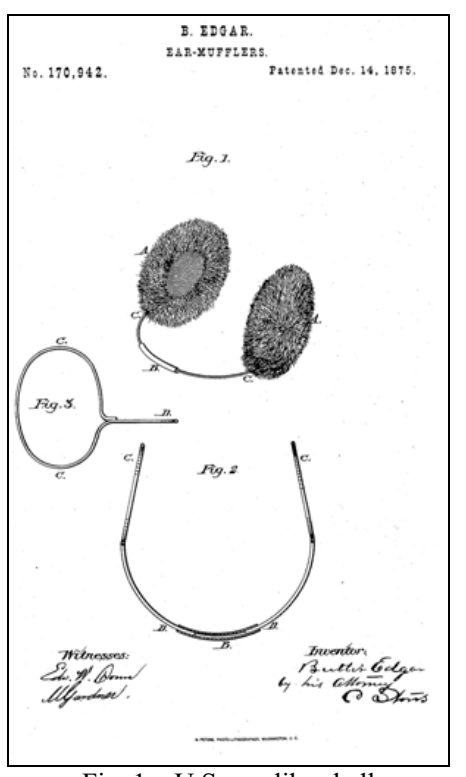

Fig. 1 - U.S. ear-like shell

The design of PPE for individual isolate agents does not represent the state of the art in PPE's. This corresponds to special projects for well-defined activities, such as those performed by astronauts or cosmonauts. The PPE used in these activities are integrated with each other and specified to protect simultaneously the worker from the many aggressive agents. The astronaut suit should protect against decompression, protect against solar radiation, ensure the survival and make easy the use of tools. The ORLAN space suit is a reference in state of the art of PPEs. It is in use on the ISS - International Space Station for extravehicular activities (Figure 2).

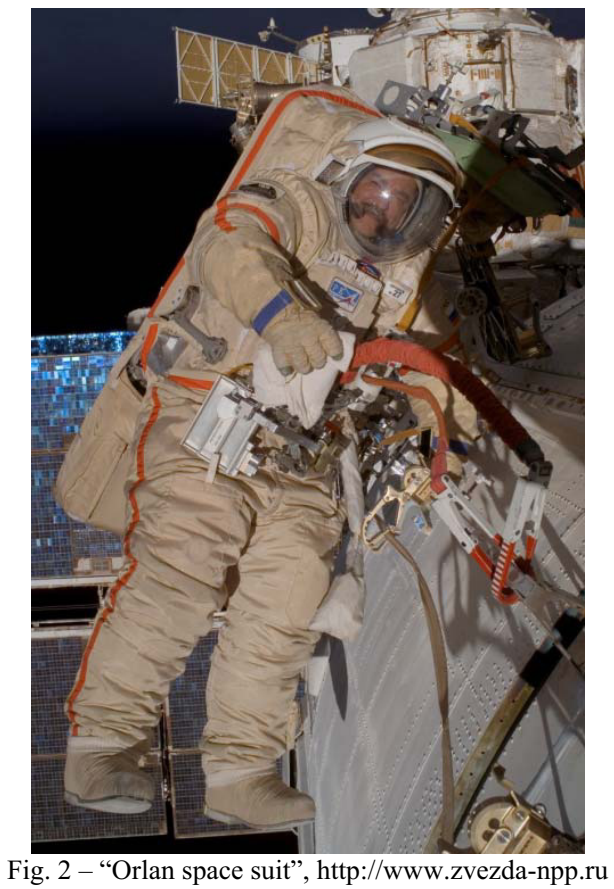

Considering the design by isolate agents in the majority of PPE's on sale, the study of the work or the analysis of activities is not addressed in the design practice as relevant, reflecting the products design methodologies adopted. The projects do not correspond to the state of the art, nor dispose about the users needs in their workplace, often exposed to more than one agent simultaneously. Considering that the occurrence is repeated, it should be considered if there are general conditionings for the lag between the state of the art and the state of prevailing practices in the production of PPE in Brazil, resulting in the selection of core functions related to isolates a technical conditioning.

The orientation of projects for one or a few isolate agents can be understood as a problem since the design of PPEs, as it suggests the adoption of a general assumption - that damage to workers health, in specific activities, are caused by one or few isolate agents or with few simultaneous exhibitions.

\section{Legislation}

In Brazil the Ordinance 3,214, enacted in 1978, regulates the Occupational Health, Safety and Labor Medicine, being mandatory for all private organizations. This Ordinance adds a set of regulatory standards. Standard 15 (NR-15) defines the unhealthy 
activities or operations - those that exposes workers to agents that might generate "damage the worker health during his working life." The objective of the ordinance seems to be to stimulate financially the employers to control hygiene in workplaces, in order to preserve workers health. The amount to be paid monthly to each employee as an additional to the possible health hazards, worth around 10,20 or $40 \%$ of the minimum wage in the region in where the facility is located. This wage must be equal or higher than the national reference, from $\mathrm{R} \$ 545.00$, approximately U.S. \$ 344.22 or \$ 239.79 USD on 6/28/2011.

The use of PPE can be legally accepted as a neutralizer of unhealthy factors to which the worker is exposedto. The PPEs are typified by parts of the body at risk. And unhealthy factors are described predominantly as isolate agents against which protection is needed. The current official typologies are then composed by two sets of categories: by type of equipment and by type of protection. There is a defined association between the equipment and the body area at risk. The hazardous agents are listed in tables of unhealthy factors.

The NR-15 does not establish associations between types of PPE, which in theory would offset the risks, and activities. The NR-15, item 15.4.1.1 states that " The regional authority responsible for the worker safety and occupational health, proven the unhealthy conditions by a technical report developed a safety engineer or an occupational physician, should establish an additional wage to the workers when it is impossible to eliminate or neutralize the existent conditions".

How can we prove the above mentioned infeasibility? Regarding the EPEs, this infeasibility probably has support in its specifications. As an exemple, with the available CAs, for $160 \mathrm{db}$ noise, the PPEs minimize up to $35 \mathrm{db}$, which would not protect the worker within the legal tolerance limits. However, there are less defined situations. Who would be favored by an assessment error - a report declaring that it is infeasible to eliminate or neutralize the unhealthy when it is feasible? The employer, who will not pay for the additional health hazards. The Social Security, who will not provide special retirement. The worker, exposed to the situation by which the protection is impossible will have its health sacrificed. There is no legal requirement that the technical report (15.4.1.1) would be issued based on experiments with PPEs in real situations. Without field experiments, the determination of infeasibility will always be presumed based on the specifications stated in the CAs.
Thus, the assumption that the PPE neutralizes the unhealthy as the CA specifications for any specific situations. However the validation process adopted in Brazil by the government authorities responsible for issuing CAs determine the orientation of the project teams to develop the appropriate PPE that will undergo testing in specialized laboratories, accredited by the Ministry of Labor and Employment. The quality of PPE with issued CAs based on laboratory tests by isolated agents is not evaluated in the field situation. The specialized laboratories do not test for type of work activity, but by type of PPE. The consequences are discussed in terms of functional effectiveness of the PPE. These conditions influence both the price restriction and the development of target projects for approval in laboratory tests, as in the case of disapproval the manufacturer could not legally sell the PPE. The PPE more financially advantageous could be chosen by the employers, which encourages PPEs producers to PPEs to develop lower-cost products enabling CAs obtaining - the PPEs isolate agents.

There is an even worse situation. In case of lack of specialized laboratories, the condition for a technical disclaimer issued by the manufacturer and presented to the MTE is considered to be met. The quality of PPE with CAs issued in terms of commitment is not evaluated by the manufacturers independent laboratories, nor by concrete situations of use. There is also a legal confusion: either it is assumed that the neutralization is unfeasible or it proves by expert opinion that 15.4.1.2 the elimination or neutralization of unhealthy will be characterized by the expert assessment developed by the competent authority, which proves the inexistence of health risk to workers.

The reference to activities is limited to associations to the unhealthiness degree, but not to the PPE. The relation to PPE with unique activities, the real work, is delegated to those who purchase the PPE, the employer, without suggesting standardization of interfaces between PPEs in order to ensure simultaneous use conditions in specific activities in which more than one type of PPE is necessary.

There is a legal constrain to the accumulation of unhealthy additional from the exposure to different "factors of unhealthy" as defined by the Ordinance 3214. A worker exposed to many agents receive additional salary for one and only one of the unhealthy factors - the one with highest degree (NR-15, item 15.3). The intention of financially fostering the adoption of healthy hygiene standards is then hampered by the legal acceptance that the protection for the factor that burdens the employer is better, with no financial burden for all the synergistic effects of agents on the 
worker health. Thus the search for a solution to any synergistic effects resulting from simultaneous exposure to several agents is minimized. This search would emphasize the suitability of PPE by economic activity, rather than by single agent.

In Brazil there is also a law that disposes about workers exposed to unhealthy working conditions special pension rights. According to Decree 3048 enacted in 1999, article 64, the special retirement is due after 15, 20 or 25 years of work on "special conditions that jeopardize the worker health or physical integrity." This legislation does not considered PPE as a neutralizer agent. Still the same Decree, article 68 explains that "The same technical report which proves the exposure of worker to harmful agents should include information about the existence of collective protection technology or individual protection technology that eliminates, minimizes or control the exposure to harmful agents to the limits of tolerance in established by labor regulations". Thus, the use of PPEs can avoid the payment of special pension rights for workers.

\section{Results and discussion}

The use of PPE is considered by the Brazilian federal legislation a neutralizing potential of health hazards factors to which the worker is exposed to. This type of adicional also exists in the U.SA. In this country the treatment given to additional health hazards is similar to what happens in Brazil regarding the payment of the additional in case of public employees (environmental differential pay 5 CFR 532, appendix A). In the U.S., the PPE is not necessarily provided by the employer. The prescription of PPE that belongs, or not, to the employee is not legislated. Usually, the employer pays for the PPE (29 CFR 1910, OSHA). However, in some cases the employee must pay for its PPE, as, for example, in the case of eyeglasses.

In the case of PPE for agriculture use, the situation is even worse, as the majority of agriculture PPEs are designed for industrial use, being adapted for agriculture use.

We can see a diffused design processes with the participation of social workers and other sectors involved in socio-technical approaches, or in action research. The case of the Volvo plant in Kalmar, Sweden, opened in 1974, suggests that participatory approaches can be adopted with advantage in less complex projects, such as the PPE. Thus, the disregard for specifications could be avoided, since the design of the PPE, not only after the event, when the use of PPEs with CAs are required for the workers, under punishment pressure. The same workers who will fall sick due to PPE inadequacy for aggressive working conditions.

Therefore, the methodology and conception used in designing PPE must be reassessed. There is a need for studies on the technological development. If the current practices and protective items remain as they are, contaminations will continue, producing both acute and chronic effects on occupational health and safety.

\section{References}

[1] International Labour Office. Guidelines on occupational safety and health management, ILO-OSH 2001, Geneva, 2001

[2] Veiga et al. A contaminação por agrotóxicos e os equipamentos de proteção individual.RBSO, v.32,n.116,p.57-68, 2007

[3] Baldi, Et Al. Pesticide contamination of workers in vineyards in France. Journal of exposure analysis and Environmental Epidemiology, v.16,n.2,p.115-24, 2006

[4] Garrigou et al. Ergonomics contribution to chemical risks prevention. Applied Ergonomics, v.42, p.321-330,2011.

[5] Havenith, G. Heat Balance when wering protective clothing. Annals of Occupational Hygiene, v.43,n.5,p.289-296, 1999

[6] Tribunal Superior Do Trabalho. Enunciados do Tribunal Superior do Trabalho relativo à insalubridade número 289. Brasília, TST, 2011.

[7] US 170.942 - Improvement in ear-mufflers. October 261875

[8] ASTM F2416 - 06e1 Standard Specification for Protective Headgear Used in Electric Personal Assistive Mobility Devices. 